\title{
Marcadores nutricionales de laboratorio en la insuficiencia renal crónica. Un estudio comparativo en pacientes en diálisis peritoneal continua ambulatoria y en hemodiálisis, en Ia Unidad de Medicina Interna del HGZ No1 del IMSS, Aguascalientes 2011
}

Pontón-Vázquez C* Osorio-Hernández H**, González-Reyes NA***

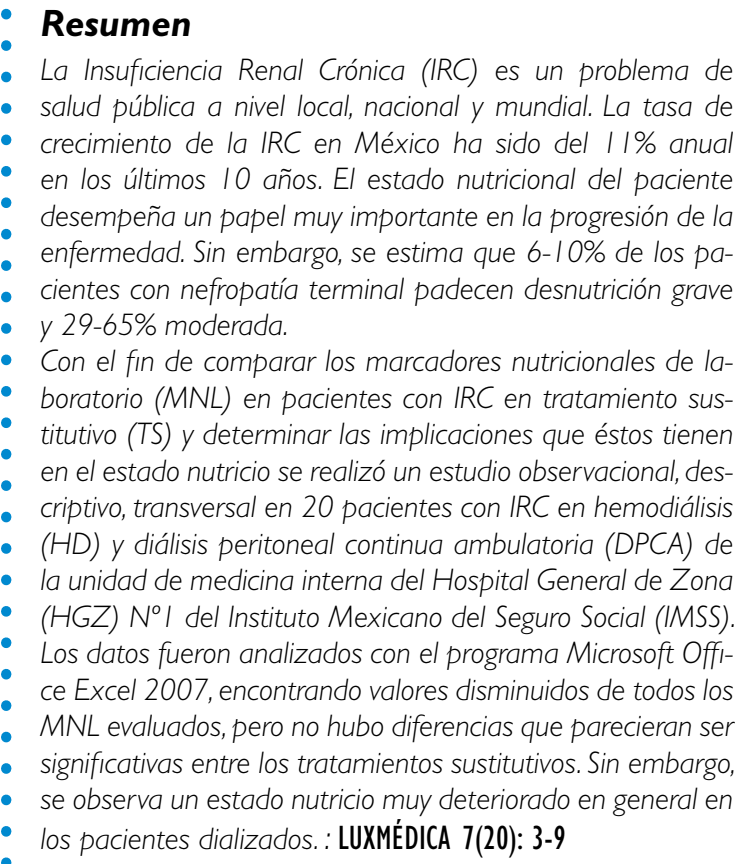

Palabras clave: insuficiencia renal crónica, diálisis peritoneal continua ambulatoria, hemodiálisis, marcadores nutricionales de laboratorio

\section{Abstract}

The Chronic Kidney Disease (CKD) is a public health problem at local, national and worldwide. The growth rate of CKD in Mexico has been $11 \%$ annual in the last 10 years. Patient's nutritional status plays an important role in disease progression. However, it's estimated that 10\% of patients with end-stage renal disease has severe malnutrition and $33 \%$ mild to moderate.

In order to compare laboratory nutritional markers (LNM) in patients with CKD in replacement therapy (RT) and to determine the implications this have in nutritional status it was performed an observational, descriptive, transversal study in 20 patients with CKD in hemodialysis and continuous ambulatory peritoneal dialysis in internal medicine unit at General Zone Hospital Nol of Mexican Institute of Social Security. Data was analyzed with Software Microsoft Office Excel 2007, finding diminished levels of all LNM evaluated, but there were no differences that seems to be significant between both replacement therapies. However, there was observed in general a much deteriorated nutritional status in dialyzed patients. LUXMÉDICA 7(20): 3-9

Key words: chronic kidney disease, continuous ambulatory peritoneal dialysis, hemodialysis, laboratory nutritional markers.

* Pasante de la Licenciatura en Nutrición de la Universidad Autónoma de Aguascalientes, Hospital General de Zona No. 1 "Dr. José Luis Ávila Pardo" del Instituto Mexicano del Seguro Social, Aguascalientes, México.

** Maestro en Ciencias de la Salud Pública, Epidemiólogo de la Clínica de Medicina Familiar Ojocaliente del Instituto de Seguridad y Servicios Sociales para los Trabajadores del Estado, Aguascalientes, México. Profesor Investigador del Departamento de Salud Pública en la Universidad Autónoma de Aguascalientes.

*** Nefróloga adscrita al Hospital General de Zona No. 1 "Dr. José Luis Ávila Pardo" del Instituto Mexicano del Seguro Social, Aguascalientes, México.

Fecha de recibido : 13 de diciembre 2011

Fecha de aceptación 9 marzo 2012

Correspondencia: Héctor Osorio Hernández, Departamento de Salud Pública del Centro de Ciencias de la Salud, Avenida Universidad 940, CP 20131. Teléfono 9108434, e mail: hosorio@correo.uaa.mx 


\section{Introducción}

La insuficiencia renal crónica (IRC) se define como el filtrado glomerular menor a $60 \mathrm{ml} / \mathrm{min}$ por un periodo de tres meses o más con la pérdida irreversible y progresiva de la función renal. ${ }^{1,2} \mathrm{La}$ IRC se ha convertido en un problema de salud pública a nivel local, nacional y mundial. La incidencia incrementada se debe, principalmente, al aumento en el número de pacientes con enfermedades crónico-degenerativas y al envejecimiento propio de la población. ${ }^{3}$ En México aproximadamente 128,000 personas padecen IRC cuya tasa de crecimiento ha sido aproximadamente del $11 \%$ anual en los últimos 10 años. ${ }^{4}$ Durante el año 2010 en el Hospital General de Zona (HGZ) No.1 del Instituto Mexicano del Seguro Social (IMSS) del estado de Aguascalientes se atendieron 693 pacientes en hospitalización con IRC. ${ }^{5}$

La tasa de mortalidad en los pacientes en diálisis es muy elevada. Además de factores de riesgo como enfermedades cardiovasculares, edad avanzada, diabetes mellitus, hipertensión arterial sistémica (HAS), dislipidemia, inflamación crónica y disfunción inmune, la desnutrición calórico-proteica se ha correlacionado fuertemente con el riesgo de mortalidad. ${ }^{1,6-18}$ Su prevalencia en pacientes en tratamiento dialítico es muy elevada variando entre $29-65 \%$ la moderada y $6-10 \%$ la severa. ${ }^{6,8,13}$ Se ha señalado que el principal inconveniente de la diálisis peritoneal (DP) es la pérdida proteica. ${ }^{6}$ Durante un régimen usual de diálisis peritoneal continua ambulatoria (DPCA), las pérdidas diarias varían de 5-15g, ${ }^{6,14}$ de las cuales 50-80\% corresponden a la albúmina. Las pérdidas de aminoácidos en DP se asemejan a la pérdida semanal en hemodiálisis (HD). Alrededor del 30\% de los aminoácidos perdidos son esenciales. ${ }^{6}$ Por su parte, la HD es un proceso catabólico con pérdida de hasta 10-13g de aminoácidos diarios. ${ }^{14}$ Alrededor del $50 \%$ de las muertes en pacientes en tratamiento sustitutivo (TS) son debidas a complicaciones cardiovasculares. ${ }^{17,15,19,20-22}$ Esto se asocia a datos antropométricos y bioquímicos de desnutrición. ${ }^{10,13,15}$ La IRC induce también un estado clínico de inmunodeficiencia con una elevada incidencia de infecciones y alta tasa de mortalidad. ${ }^{23}$ La desnutrición conduce al deterioro de la función inmune, primero a nivel celular y subsecuentemente humoral. ${ }^{24}$ Está demostrado que el tratamiento dietético en el paciente con IRC es una parte medular del manejo integral y que retarda la progresión del daño renal y mejora la calidad de vida. ${ }^{25}$ El objetivo de este estudio fue comparar los MNL en pacientes con IRC en DPCA y en HD y determinar las implicaciones que estos tratamientos tienen en el estado nutricio del paciente. 


\section{Material y métodos}

Se realizó un estudio observacional, descriptivo, transversal en el que se compararon los marcadores nutricionales de laboratorio $(\mathrm{MNL})$ de 20 pacientes con IRC en TS. Se tomó una muestra de 35 pacientes con IRC en TS mayor a 3 meses hospitalizados en la unidad de medicina interna del Hospital General de Zona (HGZ) No. 1 "Dr. José Luis Ávila Pardo" del IMSS del estado de Aguascalientes, México en el periodo abril-junio 2011. 21 pacientes pertenecían al programa de DPCA y 14 al de HD, de los cuales se eliminaron 7 y 8 respectivamente al no contar con determinaciones de albúmina séri$\mathrm{ca}$, dejando un total de 20 pacientes (14 en
DPCA y 6 en HD), 12 hombres y 8 mujeres; la edad promedio fue de 48.1 años con una mínima de 16 y máxima de 75.

Se tomaron las últimas determinaciones de hemoglobina $(\mathrm{Hb})$, hematocrito (Hcto), leucocitos, linfocitos, cuenta total de linfocitos (CTL), y creatinina y albúmina séricas, así como los principales padecimientos agregados en este grupo de población (DM2, HAS y HAS secundaria a IRC). Los datos fueron obtenidos del expediente clínico del paciente.

La base de datos fue capturada con el programa Microsoft Office Excel 2007 para la obtención de promedios, valores máximos, mínimos y prevalencias.

\section{| | | | | | | | | | | | | | | | | | | | | | | | | | | | | | | | | | | | | | | | | | | | | | | | | | | | | | | | | | | | | | | | | | | | | | | | | | | | | | | | | | | | | | | | | | | ||}

\section{Resultados}

Fueron registrados los principales MNL más recientes de 20 pacientes con IRC en TS mayor a 3 meses, así como las principales

enfermedades crónicas que acompañan a esta patología. En la tabla 1 se describen las principales características de la muestra.

\section{Tabla |}

Características generales de la muestra

\begin{tabular}{|lll|}
\hline & $\mathbf{n}$ & $\%$ \\
\hline Hombres & 12 & 60 \\
\hline$<60$ años & 12 & 60 \\
\hline DPCA & 14 & 70 \\
\hline DM2 & 9 & 45 \\
\hline HAS & 11 & 55 \\
\hline HAS sec IRC & 6 & 30 \\
\hline
\end{tabular}

DPCA: diálisis peritoneal ambulatoria continua; DM2: diabetes mellitus tipo 2; HAS: hipertensión arterial sistémica; HAS sec IRC: hipertensión arterial sistémica secundaria a insuficiencia renal crónica

Se encontró una mayor prevalencia de pacientes del sexo masculino $(60 \%)$, menores de 60 años (60\%) y en DPCA (70\%). El $45 \%$ presentaba diabetes mellitus tipo 2 (DM2) y $55 \%$ hipertensión arterial sistémica (HAS) (30\% secundaria). El 30\% mostró la presencia de ambas patologías, incrementando el riesgo cardiovascular en estos pacientes.

En la tabla 2 se presentan promedio, valor máximo y mínimo de la edad y los MNL. Los valores máximo y mínimo de todos los MNL muestran una gran variabilidad, y la mayor parte de los promedios se ubican por debajo de cifras deseables. 


\section{Tabla 2}

\section{Promedio, valor máximo y valor mínimo de las variables cuantitativas}

\begin{tabular}{|lccc|}
\hline Variable & Promedio & Máximo & Mínimo \\
\hline Edad $(\mathrm{años})$ & 48.1 & 75 & 16 \\
\hline $\mathrm{Hb}(\mathrm{g} / \mathrm{dl})$ & 9.72 & 14.2 & 6.4 \\
\hline Hcto $(\%)$ & 29.76 & 44.7 & 20.2 \\
\hline Leucocitos $(\mathrm{cel} / \mu \mathrm{l})$ & 9574.15 & 30103 & 4750 \\
\hline Linfocitos $(\%)$ & 18.2 & 35.2 & 2.6 \\
\hline CTL $($ cel $/ \mu \mathrm{l})$ & 1442 & 2860 & 500 \\
\hline Creatinina $(\mathrm{mg} / \mathrm{dl})$ & 8.74 & 18.5 & 2.7 \\
\hline Albúmina $(\mathrm{g} / \mathrm{dl})$ & 2.37 & 3.9 & 1 \\
\hline
\end{tabular}

Hb: hemoglobina; Hcto: hematocrito; CTL: cuenta total de linfocitos

Respecto a los promedios de los MNL de acuerdo al TS se encontró que la $\mathrm{Hb}$, porcentaje de linfocitos, CTL y creatinina se encuentran más bajos en los pacientes de HD que en los de DPCA $(9.61 \mathrm{~g} / \mathrm{dl}$ vs $9.77 \mathrm{~g} / \mathrm{dl}, 17.61 \%$ vs $18.5 \%, 1360 \mathrm{cel} / \mu \mathrm{l}$ vs $1477.14 \mathrm{cel} / \mu \mathrm{l}$ y $6.03 \mathrm{mg} / \mathrm{dl}$ vs $9.91 \mathrm{mg} / \mathrm{dl}$, respectivamente), mientras que lo contrario ocurre para el Hcto ( $29.71 \%$ vs $29.88 \%$ ), leucocitos (8809.28cel/ $\mu$ l vs $11358.83 \mathrm{cel} /$ $\mu \mathrm{l})$ y albúmina $(2.34 \mathrm{~g} / \mathrm{dl}$ vs $2.46 \mathrm{~g} / \mathrm{dl})$. Respecto a los valores máximo y mínimo destaca la albúmina al encontrarse ambos valores de los pacientes en DPCA por debajo de los que presentan los pacientes en HD (tabla 3).

\section{Tabla 3}

\section{Comparativo de promedio, valor máximo y valor mínimo de acuerdo con la modalidad de diálisis}

\begin{tabular}{|lcccccc|}
\hline Variable & \multicolumn{2}{c}{ Promedio } & \multicolumn{2}{c}{ Máximo } & \multicolumn{2}{c|}{ Mínimo } \\
\hline & DPCA & HD & DPCA & HD & DPCA & HD \\
\hline $\mathrm{Hb}(\mathrm{g} / \mathrm{dl})$ & 9.77 & 9.61 & 13.4 & 14.2 & 6.7 & 6.4 \\
\hline $\mathrm{Hcto}(\%)$ & 29.71 & 29.88 & 40 & 44.7 & 21.4 & 20.2 \\
\hline Leucocitos $(\mathrm{cel} / \mu \mathrm{l})$ & 8809.28 & 11358.83 & 15390 & 30103 & 4800 & 4750 \\
\hline Linfocitos $(\%)$ & 18.5 & 17.61 & 35.2 & 32.6 & 4.8 & 2.6 \\
\hline $\mathrm{CTL}(\mathrm{cel} / \mu \mathrm{l})$ & 1477.14 & 1360 & 2860 & 2260 & 500 & 660 \\
\hline Creatinina $(\mathrm{mg} / \mathrm{dl})$ & 9.91 & 6.03 & 18.5 & 12.9 & 2.7 & 3.3 \\
\hline Albúmina $(\mathrm{g} / \mathrm{dl})$ & 2.34 & 2.46 & 3.4 & 3.9 & 1 & 1.6 \\
\hline
\end{tabular}

DPCA: diálisis peritoneal continua ambulatoria; HD: hemodiálisis; Hb: hemoglobina; Hcto: hematocrito; CTL: cuenta total de linfocitos

En la tabla 4 se resumen las frecuencias y porcentajes de los rangos de referencia deseables y de aquellos que suponen un ma- yor riesgo. Fueron considerados los cuatro MNL más comúnmente utilizados: $\mathrm{Hb}$, $C T L$, y creatinina y albúmina séricas. 


\section{Tabla 4}

Frecuencia y porcentaje de pacientes dentro y por debajo de rangos de referencia deseables para pacientes en diálisis de los MNL más significativos para evaluación nutricia

\begin{tabular}{|c|c|c|c|c|c|c|}
\hline \multirow[t]{2}{*}{ Variable } & \multicolumn{2}{|c|}{ Total } & \multicolumn{2}{|c|}{ DPCA } & \multicolumn{2}{|c|}{ HD } \\
\hline & $\mathbf{n}$ & $\%$ & $\mathbf{n}$ & $\%$ & $\mathbf{n}$ & $\%$ \\
\hline \multicolumn{7}{|l|}{$H b(g / d l)$} \\
\hline $12-16$ & 2 & 10 & 1 & 7.1 & 1 & 16.7 \\
\hline$<11$ & 15 & 75 & 10 & 71.4 & 5 & 83.3 \\
\hline \multicolumn{7}{|l|}{ CTL (cel/ $\mu \mathrm{l})$} \\
\hline $1500-4000$ & 9 & 45 & 6 & 42.8 & 3 & 50 \\
\hline$<900$ & 4 & 20 & 2 & 14.3 & 2 & 33.3 \\
\hline \multicolumn{7}{|c|}{ Creatinina (mg/dl) } \\
\hline $9-11$ & 4 & 20 & 4 & 28.6 & 0 & 0 \\
\hline$<7.5$ & 8 & 40 & 3 & 21.4 & 5 & 83.3 \\
\hline \multicolumn{7}{|c|}{ Albúmina (g/dl) } \\
\hline $3.5-5$ & 1 & 5 & 0 & 0 & 1 & 16.7 \\
\hline $3-3.4$ & 4 & 20 & 3 & 21.4 & 1 & 16.7 \\
\hline$<3$ & 15 & 75 & 11 & 78.6 & 4 & 66.7 \\
\hline
\end{tabular}

DPCA: diálisis peritoneal continua ambulatoria; HD: hemodiálisis; Hb: hemoglobina; CTL: cuenta total de linfocitos

Respecto a la $\mathrm{Hb}$, sólo el $10 \%$ de los pacientes se encontró en rango de normalidad. El $75 \%$ presentó valores menores a $11 \mathrm{~g} / \mathrm{dl}$, representando un mayor porcentaje en los pacientes en HD. En cuanto a la CTL se ubicó al $45 \%$ de la muestra en rango de normalidad de 1500 a 4000cel/ $\mu \mathrm{l}$, mientras que $20 \%$ mostró valores por debajo de $900 \mathrm{cel} / \mu \mathrm{l}$, siendo un mayor porcentaje entre los pacientes en HD. La creatinina sérica se encontró baja $(<7.5 \mathrm{mg} / \mathrm{dl})$ en $40 \%$ de los pacientes con mayor prevalencia entre los hemodializados. El $20 \%$ estuvo dentro de los valores esperados (9-11 mg/dl). La albúmina sérica por otra parte se observa normal en sólo $5 \%$ de los pacientes, $20 \%$ presenta una depleción moderada (3-3.4g/dl) y $75 \%$ depleción severa $(<3 \mathrm{~g} / \mathrm{dl})$. Estos valores bajos se encontraron en mayor porcentaje en los pacientes en DPCA.

\section{| | | | | | | | | | | | | | | | | | | | | | | | | | | | | | | | | | | | | | | | | | | | | | | | | | | | | | | | | | | | | | | | | | | | | | | | | | | | | | | | | | | | | | | | | | | | | | | | | | | | | | | | | | | | | | |}

\section{Discusión}

Diversos estudios han evaluado varios parámetros de laboratorio en pacientes con IRC en TS con la finalidad de identificar aquellos que predisponen a una mayor morbi-mortalidad cuando sus valores se encuentran alterados. Dos de los principales marcadores evaluados son la albúmina y creatinina séricas al ser buenos indica- dores de la evolución clínica del paciente, además de ser confiables para la evaluación nutricia. Avram et.al. ${ }^{17}$ encontraron promedios de albúmina y creatinina de $3.6 \mathrm{~g} / \mathrm{dl}$ y $11.89 \mathrm{mg} / \mathrm{dl}$ respectivamente en pacientes en DPCA. Af_ar, et.al. ${ }^{16}$ hallaron valores muy similares para la albúmina sérica $(3.7 \mathrm{~g} / \mathrm{dl})$. En el presente estudio, estos marcadores en el mismo grupo de 
población se encuentran muy por debajo $(9.91 \mathrm{mg} / \mathrm{dl}$ para la creatinina y $2.34 \mathrm{~g} / \mathrm{dl}$ para la albúmina).

Por su parte, De Araujo et.al. ${ }^{7}$ observaron promedios de creatinina de $8.9 \mathrm{mg} / \mathrm{dl}$ y de albúmina de $3.8 \mathrm{~g} / \mathrm{dl}$ en pacientes en $\mathrm{HD}$, mientras que Hrnciarikova, et.al. ${ }^{24}$ encontraron valores de $10 \mathrm{mg} / \mathrm{dl}$ de creatinina y $3.6 \mathrm{~g} / \mathrm{dl}$ de albúmina, en contraste con los obtenidos en este estudio de $6.03 \mathrm{mg} / \mathrm{dl}$ y de $2.46 \mathrm{~g} / \mathrm{dl}$, respectivamente.

En una investigación realizada en población mexicana por Méndez Durán et.al. ${ }^{25}$ en la que se compararon los niveles de albúmina sérica en pacientes en DP y HD se encontraron cifras de $2.8 \mathrm{~g} / \mathrm{dl}$ y $3 \mathrm{~g} / \mathrm{dl}$ respectivamente, sin diferencias estadísticamente significativas. Af_ar,et.al. ${ }^{16}$ por otra parte, que estudiaron pacientes en DPCA, encontraron cifras de $\mathrm{Hb}$ de $10.7 \mathrm{~g} / \mathrm{dl}$, mientras que las observadas por Hrnciarikova, et.al. ${ }^{24}$ en pacientes en HD fueron de $9.2 \mathrm{~g} / \mathrm{dl}$. Los hallazgos del presente estudio se ubicaron en $9.77 \mathrm{~g} / \mathrm{dl}$ y $9.61 \mathrm{~g} / \mathrm{dl}$ respectivamente. Finalmente, Hrnciarikova, et.al. ${ }^{24}$ analizaron también los valores de CTL en HD encontrando cifras promedio de $1398.3 \mathrm{cel} /$ $\mu \mathrm{l}$, muy similares a las observadas en este estudio de $1360 \mathrm{cel} / \mu \mathrm{l}$.

Aunque no se encontró mucha diferencia entre los tratamientos dialíticos, se observa que la mayor parte de los pacientes evaluados presenta depleción importante de los $M N L$, destacando la hemoglobina y la albúmina sérica al ubicar a una proporción muy grande de pacientes en valores subóptimos. La mayor parte de los pacientes en HD se encontró por debajo de los rangos deseables tanto de $\mathrm{Hb}$ como de CTL y creatinina, mientras que todos los pacientes en DPCA mostraron valores disminuidos de albúmina sérica. Esto podría ser sugestivo de una mayor prevalencia de desnutrición proteica entre los pacientes en DPCA, sin embargo, se requiere de más investigación al respecto, sobre todo a largo plazo.

\section{Conclusiones}

El estado nutricio del paciente con IRC en TS es un factor clave en la evolución de la enfermedad, un buen manejo nutricio se ve reflejado principalmente en los resultados de laboratorio. Por diversas razones, el paciente nefrópata en DPCA o en HD se desnutre mostrando una depleción importante en sus marcadores nutricionales, en este estudio se encontraron valores disminuidos de todos los MNL evaluados, pero no hubo diferencias que parecieran ser significativas entre los dos tratamientos sustitutivos. Sin embargo, se observa un estado nutricio muy deteriorado en general en los pacientes dializados. Esto sugiere que debe ponerse mayor énfasis en el tratamiento nutricio del enfermo renal, principalmente en lo concerniente al aporte proteico para reponer las pérdidas intradialíticas en ambas modalidades de diálisis.

\section{Bibliografía}

1. Osuna Ortega A Mataix Verdú J. Enfermedades renales. En: Mataix Verdú J. Tratado de nutrición y alimentación, vol. II; Situaciones fisiológicas y patológicas. Barcelona, Océano/Ergon, 2009, pág. 17001709.

2. Ramiro $M$ Lifshitz A Halabe J Frati A. El internista. $3^{a}$ edición. México, Nieto Editores, 2008, pág. 544556.
3. Méndez Durán A. Prevención del daño, manejo de la Enfermedad Renal Crónica en el primer nivel de atención médica. Aten Fam [Revista en línea] 2010 [citado 12 de junio 2011];17(3):[aprox 9 pantallas]. Disponible en: http://www.journals.unam.mx/index.php/raf/article/view/19296

4. Fundación Mexicana del Riñón, A.C [sede Web]. México: FMR; 2005 [acceso 12 junio 2011]. Estadísticas [aprox 2 pantallas]. Disponible en: http://www. fundrenal.org.mx/home.html 
5. Instituto Mexicano del Seguro Social, Hospital General de Zona No.1 "Dr. José Luis Ávila Pardo", Aguascalientes, México, Sistema Médico Operativo (SIMO). Concentrado de informes 2010 [acceso 6 junio 2011].

6. Martins C Riella M. Nutrición y hemodiálisis. Martins C Pecoits Filho R Riella $M$. Nutrición y diálisis peritoneal. En: Riella $M$, Martins $C$. Nutrición y riñón. $2^{a}$ reimpresión, Buenos Aires: Médica Panamericana; 2007, pág. 124-128, 144-148.

7. De Araújo IC, Kamimura MA, Draibe SA, Fernandes Canziani ME, Manfredi SR, Avesani CM, et al. Nutritional parameters and mortality in incident hemodialysis patients. J Ren Nutr [Revista en línea] 2006 [citado 22 junio 2011];16(1):[aprox 22 pantallas]. Disponible en: http://www.jrnjournal.org/article/ S1051-2276(05)00174-3/fulltext

8. González MT, Arango JJ. Concordancia entre IMC y albúmina como método diagnóstico de desnutrición en pacientes en diálisis. Acta Med Colomb [Revista en línea] 2006 [citado 25 marzo 2011];31(2):[aprox 9 pantallas]. Disponible en: http://www.actamedcolomb.org.co/vol31-02/pdf/b1.pdf

9. Bravo Ramírez AM, Chevaile Ramos A, Hurtado Torres GF. Composición corporal en pacientes con insuficiencia renal crónica y hemodiálisis. Nutr Hosp [Revista en línea] 2010 [citado 11 marzo 2011];25(2):[aprox 9 pantallas]. Disponible en: www.nutricionhospitalaria.com/pdf/4282.pdf

10. Kuhlmann MK, Levin NW. How Common Is Malnutrition in ESRD? New Approaches to Diagnosis of Malnutrition. Blood Purif [Revista en línea] 2008 [citado 22 junio 2011];26:[aprox 9 pantallas]. Disponible en: http://content.karger.com/produktedb/ produkte. asp?typ $=$ pdf \&file $=000110564$

11. National Kidney Foundation. K/DOQI nutrition in chronic renal failure. Am J Kidney Dis [Revista en línea] 2000 [citado 21 mayo 2011];35(6)Suppl 2:[aprox 381 pantallas]. Disponible en: http://www. kidney.org/professionals/kdoqi/pdf/KDOQI2000NutritionGL.pdf

12. Chung $S H$, Stenvinkel $P$, Lindholm $B$, Avesani $C M$. Identifying and managing malnutrition stemming from different causes. Perit Dial Int [Revista en línea] 2007 [citado 22 junio 2011];27(Suppl 2):[aprox 11 pantallas]. Disponible en: http://www.pdiconnect. com/cgi/reprint/27/Supplement_2/S239

13. Palomares Bayo $M$, Oliveras López MJ, Osuna Ortega A, Asensio Peinado C, Quesada Granados JJ, López García de la Serrana H, et al. Evolución de parámetros bioquímicos nutricionales en pacientes de hemodiálisis durante un año de seguimiento. Nutr Hosp [Revista en línea] 2008 [citado 25 marzo 2011];23(2): [aprox 14 pantallas]. Disponible en: http://scielo.isciii.es/pdf/nh/v23n2/original8.pdf

14. Bowling T. Apoyo nutricional para niños y adultos, manual para la práctica hospitalaria. México, Mc Graw-Hill, 2006, pág. 100, 102.

15. Palomares Bayo M, Quesada Granados JJ, Osuna Ortega A, Asensio Peinado C, Oliveras López MJ, López $\mathrm{G}$ de la Serrana $\mathrm{H}$, et al. Estudio longitudinal del Índice de masa corporal (IMC) en pacientes en diálisis. Nutr Hosp [Revista en línea] 2006 [citado 24 marzo 2011];21(2):[aprox 15 pantallas]. Disponible en:http://scielo.isciii.es/pdf/nh/v21n2/original5.pdf

16. Af ar B, Sezer S, Ozdemir FN, Celik H, Elsurer R, Haberal $M$. Malnutrition-inflammation score is a useful tool in peritoneal dialysis patients. Perit Dial Int [Revista en línea] 2006 [citado 22 junio 2011];26: [aprox 13 pantallas]. Disponible en: http://www.pdiconnect.com/cgi/reprint/26/6/705

17. Avram MM, Fein PA, Rafiq MA, Schloth T, Chattopadhyay J, Mittman N. Malnutrition and inflammation as predictors of mortality in peritoneal dialysis patients. Kidney international [Revista en línea] 2006 [citado 22 junio 2011];70(104 suppl):[aprox 7 pantallas]. Disponible en: http://www.nature.com/ki/ journal/v70/n104s/pdf/5001968a.pdf

18. Prasad N, Gupta A, Sharma RK, Sinha A, Kumar R. Impact of nutritional status on peritonitis in CAPD patients. Perit Dial Int [Revista en línea] 2007 [citado 22 junio 2011];27:[aprox 11 pantallas]. Disponible en: http://www.pdiconnect.com/cgi/reprint/27/1/42.pdf

19. Escott-Stump $S$. Nutrición, diagnóstico y tratamiento. $5^{\mathrm{a}}$ ed. México: Mc Graw-Hill; 2005.

20. Carrero JJ, Qureshi AR, Axelsson J, Avesani CM, Suliman $M E$, Kato $S$, et al. Comparison of nutritional and inflammatory markers in dialysis (HD) patients with reduced appetite. Am J Clin Nutr [Revista en línea] 2007 [citado 21 junio 2011]; 85: [aprox 13 pantallas]. Disponible en: www.ajcn.org/content/85/3/695. full.pdf

21. Kaysen GA. Biochemistry and biomarkers of inflamed patients: why look, what to assess. Clin J Am Soc Nephrol [Revista en línea] 2009 [citado 3 abril 2011];4 Suppl 1:[aprox 15 pantallas]. Disponible en: http://cjasn.asnjournals.org/content/4/Supplement_1/S56.full.pdf

22. Kaysen GA. Association between inflammation and malnutrition as risk factors of cardiovascular disease. Blood Purif [Revista en línea] 2006 [citado 22 junio 2011];24:[aprox 9 pantallas]. Disponible en: http:// content.karger.com/produktedb/produkte.asp?typ $=$ pdf\&file $=$ BPU2006024001051

23. Sayarlioglu $H$, Erkoc R, Demir C, Dogan E, Sayarlioglu $M$, Oner $A F$, et al. Nutritional status and immune functions in maintenance hemodialysis patients. Mediators Inflamm [Revista en línea] 2006 [citado 6 julio 2011]; 202:[aprox 7 pantallas]. Disponible en: http://downloads.hindawi.com/journals/ $\mathrm{mi} / 2006 / 020264 . p d f$

24. Hrnciarikova D, Juraskova B, Zadak Z, Hronek M. Present state of evaluating malnutrition in the elderly-analysing indicators. Biomed Pap Med Fac Univ Palacky Olomouc Czech Repub [Revista en línea] 2006 [citado 6 julio 2011];150(2):[aprox 9 pantallas].

Disponible en: http://biomed.papers.upol.cz/savepdfs/bio/2006/02/03.pdf

25. Méndez Durán A, Méndez Durán E, Pérez García F, Esparza Bustos MS, Espinosa Furlong MC. Albúmina sérica como factor pronóstico de mortalidad en pacientes con diálisis, análisis comparativo. Revista de Especialidades Médico-Quirúrgicas [Revista en línea] 2002 [citado 25 marzo 2011];7(1):[aprox 9 pantallas]. Disponible en: http://redalyc.uaemex.mx/redalyc/pdf/473/47370105.pdf 